\title{
Theoretical and practical application of Wang-Müller line generalisation algorithm
}

\author{
Motiejus Jakštys ${ }^{\mathrm{a}}$, and Tomas Straupis ${ }^{\mathrm{b}}$ * \\ ${ }^{a}$ Vilnius University,motiejus@jakstys.lt \\ ${ }^{b}$ Association Atvirasis žemèlapis, tomasstraupis@gmail.com* Corresponding author
}

Keywords: spatial data, cartographic generalisation, multiple representation, multi scale maps

\begin{abstract}
:
Expectation and demand by map users is calling for a much more frequent updates to maps. If just some decades ago it was acceptable to update maps once in 5 to 10 years, it is today expected that maps in all scales are updated at least once a month (which is the case for Lithuanian NMA national dataset - GRPK). In some cases map needs to be updated as soon as new data is collected, which in the rapid rise of the usage of remote sensing technologies and participatory on-line mapping (like OpenStreetMap) means almost real-time updates. The need for good cartographic algorithms of automatic generalisation is rising rapidly. With the absence (or low availability) of such algorithms, cartographic generalisation is usually skipped.
\end{abstract}

There are a lot of different generalisation operators which need open implementations, this work is about line generalisation (which can also be used for polygon generalisation). Two algorithms usually used for this task are Douglas Peucker and Visvalingam Wyatt. Unfortunately these algorithms are purely geometric ones and they do not fulfil cartographic requirements, i.e. a human cartographer would generalise the lines in a different way. For natural features such as rivers and coastlines it is important to preserve characteristic properties of the features being generalised, namely the bendiness as a whole and specific important bends in particular - as those are the features which give understanding on the characteristics of the river: is it flowing through plains or down the mountain, size of debit etc. If a river is nearly straight, it should remain such after simplification. An overly straightened river will look like a canal, and the other way around - too curvy would not reflect the natural shape. Conversely, if the river originally is highly wiggly, the number of bends should be reduced, but not removed altogether. Natural line simplification problem can be viewed as a task of finding a delicate balance between two competing goals:

- Reduce detail by removing or simplifying "less important" features.

- Retain enough detail, so the original is still recognizable.

Given the discussed complexities with natural features, a fine line between under-simplification (leaving an object asis) and over-simplification (making a straight line) needs to be found. Therein lies the complexity of simplification algorithms: all have different trade-offs.

Scientific relevance of this work - the simplification processes (steps) described by the Wang-Müller algorithm — are analysed in detail, practically implemented, and the implementation is described. That expands the knowledge of cartographic theory about the generalisation of natural objects' boundaries after their natural defining properties.

In the original Wang-Müller article introducing the algorithm, the steps are not detailed in a way that can be put into practice for specific data (Zeshen Wang, Jean-Claude Müller, 1998); the steps are specified in this work. Practically, this work makes it possible to use open-source software to perform cartographic line generali sation. The developed specialised cartographic line simplification algorithm can be applied by cartographers to implement automatic data generalisation solutions. Given the open-source nature of this work, the algorithm implementation can be modified freely.

Having an open and very well described implementation of such algorithms is essential for rising generation of cartographers, because without in-depth knowledge of the inners of such algorithms they would be restricted to blind usage of closed algorithms and will therefore lack actual understandings of what is being done and most importantly why.

The purpose of the work is to implement a cartographic line generalisation algorithm on the basis of Wang-Müller algorithm, using open-source software. Tasks: 
- Identify main river generalisation problems, using classical line simplification algorithms.

- Define the method of the Wang-Müller technical implementation.

- Implement Wang-Müller algorithm technically, explaining the geometric transformations in detail.

- Apply the created algorithm for different datasets and compare the results with national datasets.

Up until this moment the algorithm has been described in more detail and most of it is implemented:

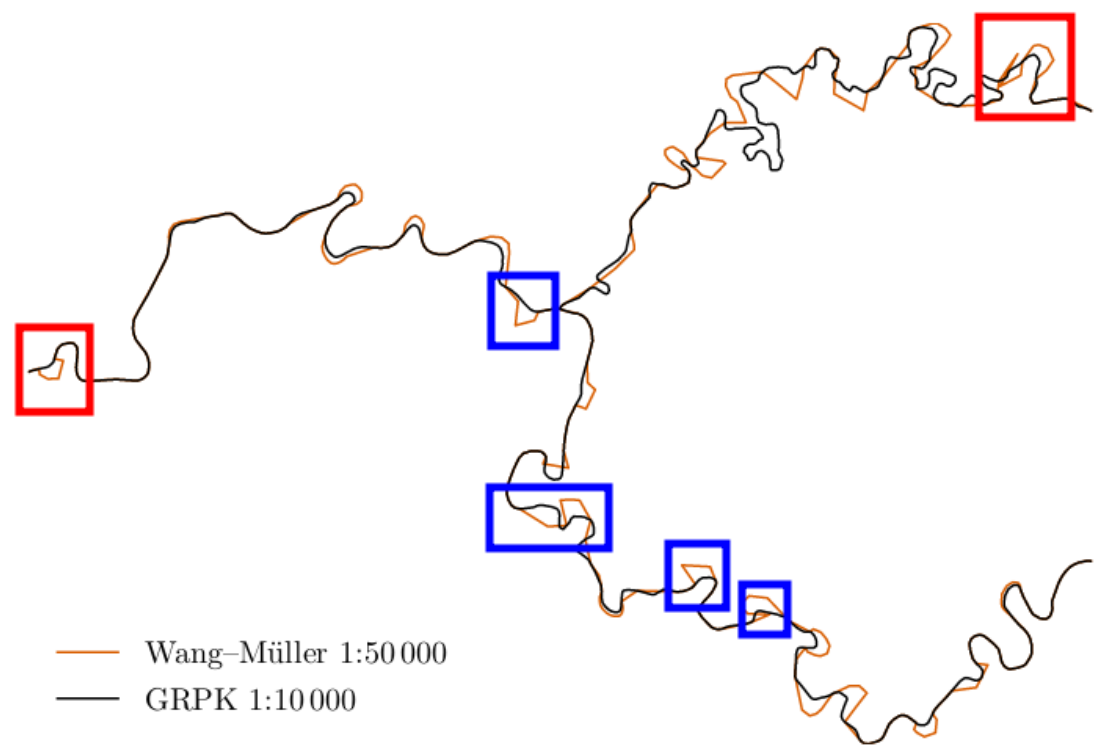

Figure 1. Current Wang-Müller generalisation example with remaining errors marked in red and imperfect segments marked in blue.

However some work needs to be done for this algorithm to be usable in practice:

- Bend combination has to be implemented - when two similar bends are one next to another (and they are small), they have to be replaced with one slightly larger bend thus reducing clutter but leaving essential characteristic - bend.

- Reasons for resulting errors and quirks (examples depicted in Fig. 1) have to be identified, analysed and implementation improved.

- Some aspects of algorithm have to be analysed, tested and described, to name a few:

$\circ \circ$ Should algorithm always remove only the smallest bend in an iteration or can it go through a number of bends in one iteration?

$\circ \circ$ How bend manipulation (exaggeration, elimination) influences neighbouring bends and what impact does it have to the final result?

Final expected results are:

- a document describing all aspects of this algorithm - allowing for other researchers to follow up as well as for cartography students to better understand the intricacies of the algorithm

- $\quad$ open source implementation of the algorithm using open technologies - allowing anybody to use the algorithm thus improving the cartographic results of medium to small scale maps.. 